\title{
RHEUMATOLOGY
}

\section{The adolescent with rheumatic disease}

\author{
R Sathananthan, J David
}

This is the sixth article in a series on rheumatology.
Royal Berkshire and Battle Hospitals NHS Trust, Reading, Berkshire

R Sathananthan J David

Correspondence to: Dr J David, Royal Berkshire and Battle Hospitals NHS

Trust, Oxford Road,

Reading, Berkshire RG3 1AG.
Adolescence is the period between childhood and adulthood when major biological and psychosocial development occurs. During childhood, toddlers are initially wholly dependent on their parents to meet their everyday needs, such as washing, dressing, and feeding. As the child grows, so the parental role needs to accommodate these changes.

Puberty results in skeletal growth, maturation, and the development of secondary sex characteristics. Teenagers are extremely sensitive to the opinions of their peers and will often compare themselves with others. Even the perception, real or otherwise, of underdevelopment, obesity, or acne can lead to feelings of inferiority, low esteem, and loss of confidence. Awareness of self and body image, as well as a concept of sexuality, becomes important. Seeking friends and sexual relationships, plans for the future, and career choices need to be made. There is an increasing need for independence and a separate identity from the parents. Adulthood signifies this independenceoften the completion of education, starting of employment, financial independence, leaving home, and the possibility of making a contribution to society.

Adolescents with chronic illness and disability may be more dependent on their parents for support. They may also feel overprotected, sheltered, and unable to make their own decisions. They may sense a general lack of control in their lives as they struggle to seek their own independence. Self consciousness about physical appearance often leads to withdrawal and an inability to socialise. Frequent admissions to hospital contribute to social isolation and loneliness. Career opportunities may be limited further increasing reliance on the family and community for financial support.

\section{Juvenile chronic arthritis}

Although there are conditions that are exclusive to the young, most rheumatic diseases form part of a continuum with those seen in adulthood. The pattern of arthritis can sometimes be predicted, depending on the age and sex of the patient.

Juvenile chronic arthritis can be classified into three major subgroups according to type of onset. Pauciarticular arthritis is the most common and accounts for $50 \%$ of cases. Polyarticular disease accounts for $17 \%$, and systemic onset $11 \%$ of cases. ${ }^{1}$
PAUCIARTICULAR ARTHRITIS

Pauciarticular onset arthritis is essentially a disease of childhood, mostly affecting young girls. The peak age of onset is between 1 and 3 years of age, but it can present in adolescence. The most commonly affected joints are the knees and ankles and symptoms are often mild. Antinuclear antibodies are found in $40-75 \%$ of children with pauciarticular disease and their presence is strongly associated with anterior uveitis, which needs to be screened for. The prognosis of this form of arthritis is good.

\section{POLYARTICULAR DISEASE}

In polyarticular disease, approximately $10 \%$ of cases are IgM rheumatoid factor positive and are associated with HLA-DR4. ${ }^{2}$ The disease is clinically identical to adult rheumatoid arthritis, affects predominantly girls from the age of 10 years, and is characterised by symmetrical erosive small joint polyarthritis with nodule formation correlating with seropositivity. Destruction of large joints, especially hips and knees, can occur early in some. It is recognised that the presence of hip disease is an important determinant of later disability. ${ }^{3}$ Disease activity usually persists into adulthood and patients are likely to have multiple joint replacements. ${ }^{4}$ Joint destruction occurs relatively early in the disease and it is now considered that aggressive treatment should be introduced early to induce disease remission. ${ }^{5}$ Methotrexate is regarded as the drug of choice in the treatment of seropositive disease and the usual dose is in the range 5-10 mg/m $\mathrm{m}^{2}$ per week. ${ }^{6}$ The risk of cancer and infertility do not appear to be increased. ${ }^{7}$ Short term studies using hydroxychloroquine and penicillamine have shown no therapeutic effect. ${ }^{8}$ A few patients have been shown to respond to intramuscular gold, reflecting the similarities with adult onset rheumatoid arthritis. ${ }^{9}$

SYSTEMIC ONSET ARTHRITIS

Systemic onset juvenile chronic arthritis occurs infrequently in adolescence and affects both sexes equally. The disease is characterised by typical spiking fevers, an evanescent rash, lymphadenopathy, hepatosplenomegaly, and a polyserositis. Arthritis may not be a feature at presentation and can sometimes develop months after the onset of systemic symptoms. Occasionally a progressive destructive arthritis can occur. The disease is both antinuclear antibody and rheumatoid factor negative. Although non-steroidal anti-inflammatory 
drugs have an important role in the treatment of systemic disease, some patients need corticosteroid treatment, which may lead to osteoporosis, growth retardation, and truncal obesity. The important consequence of this, particularly in young girls concerned about their appearance and body image, is that they may not comply with medication, resulting in failure to control disease activity.

Contrary to common belief that children grow out of their arthritis with little deformity, several studies have shown that at 10 years, between $9 \%$ and $48 \%$ of patients have severe functional limitation and approximately 50\% have active disease as they enter adult life. ${ }^{5}$ Delayed puberty and coexisting growth retardation may make adolescents with arthritis appear younger than they are, which can lead to further embarrassment about being treated as a child. Anxiety and depression feature highly in adolescents with juvenile chronic arthritis. Short stature and restricted mobility are considered key risk factors in psychological morbidity. ${ }^{10}$ However, it is important to recognise that the age at onset of arthritis can affect the way the child copes psychologically. Thus young children with arthritis may become better adjusted adolescents because they cannot remember being normal. ${ }^{4}$

\section{ANKYLOSING SPONDYLITIS}

Juvenile onset ankylosing spondylitis usually presents in adolescence and more commonly affects boys. Their symptoms may first present in childhood with a large joint arthritis, frequently affecting the lower limb such that the condition may be misdiagnosed as pauciarticular juvenile chronic arthritis. Axial disease and sacroiliitis may develop as late as $5-10$ years after the onset of symptoms. ${ }^{11}$ In these individuals there is an association with HLA B27, but IgM rheumatoid factor and antinuclear antibodies are negative. The presence of an enthesitis increases the possibility of an HLA B27 associated disorder, as does a family history of spondyloarthropathy and attacks of acute iritis. These individuals may have persistent or recurrent bouts of arthritis and enthesitis resulting in severe disability due to foot and hip involvement, regardless of the intensity of the axial disease.

JUVENILE PSORIATIC ARTHRITIS

In patients with juvenile psoriatic arthritis, the typical rash may not be present. In such cases the presence of dactylitis, nail changes, or a family history of psoriasis may aid in making the diagnosis. ${ }^{12}$ A small percentage of children develop enthesitis, sacroiliitis, and back pain, but the frequency is much lower than in those with juvenile ankylosing spondylitis and the incidence of HLA B27 is only slightly increased. ${ }^{11}$ The disease may have a pauciarticular onset in children, and then develop into an asymmetrical destructive polyarthritis in adolescence. It is important to consider aggressive treatment early before irreversible joint damage occurs, and in this group methotrexate is increasingly being used. ${ }^{13}$
Table 1 Clinical manifestations of systemic lupus erythematosus in childhood (from reference 15)

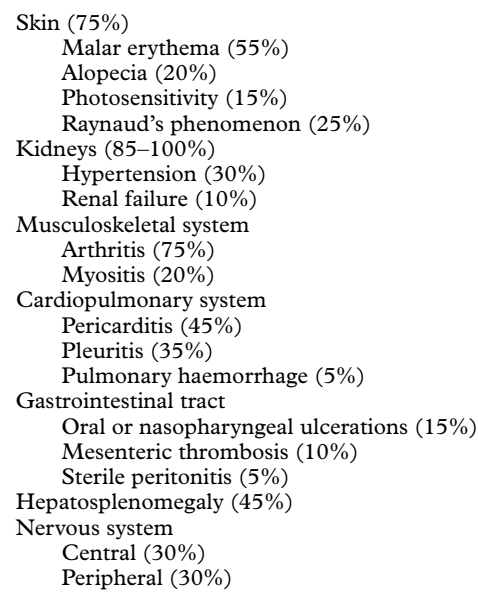

Inflammatory connective tissue diseases Approximately $20 \%$ of all cases of systemic lupus erythematosus (SLE) occur before the age of 18 years. $^{14}$ Boys tend to be more commonly affected before the onset of puberty. Table 1 provides an outline of the clinical features of SLE in childhood and adolescence. ${ }^{15}$ Neuropsychiatric symptoms and headaches are common in cerebral lupus. Patients may present with affective disorders, mood swings, or cognitive impairment. There may be difficulty in determining whether these symptoms are due to the lupus, to the effect of treatment with steroids, or to a reactive depression. Cerebral lupus can exist in isolation or occur as part of multiorgan involvement and can present at any time during the course of the illness. Up to $15 \%$ of patients may present with thrombocytopenia, such that the initial working diagnosis may be idiopathic thrombocytopenic purpura.

Although most patients are steroid responsive, a few require more aggressive immunosuppression. The role of splenectomy is controversial in this group of patients because of the increased risk of infection. Renal involvement affects a significant number of children with lupus and the overall prognosis of these patients is related to the severity of renal disease. In nephrotic syndrome, the presence of hypertension is common and requires aggressive treatment. Current regimens involve the use of high dose steroids, azathioprine, or oral or intravenous pulse cyclophosphamide. Infertility and ovarian failure may occur as a result of cyclophosphamide treatment. Menstrual disorders are common and cumulative dose related. ${ }^{16}$ There is also an increased risk of secondary malignancy, such as non-Hodgkin's lymphoma. ${ }^{17}$ The anxiety and depression that often occur in this group of patients may be separate from any CNS involvement. Prolonged hospital admission, side affects of steroids, and growth retardation are the major contributory factors to these psychological symptoms and all need to be addressed. As in patients with systemic onset juvenile chronic arthritis, compliance with steroid treatment may be a problem. 
Scleroderma, dermatomyositis, Wegener's granulomatosis, and Takayasu's arteritis are all rare, but often make their debut in adolescence. ${ }^{18}$

\section{Non-inflammatory rheumatic disease} Non-inflammatory musculoskeletal problems are a common reason for referral in adolescence. In those who present with back pain, postural problems and hypermobility are the most likely underlying causes.

Scheuermann's osteochondritis can cause a thoracic kyphosis. ${ }^{19}$ The pain is usually aggravated by activity and relieved by rest and can be diagnosed on $x$ ray by anterior wedging by more than $5^{\circ}$ of three or more adjacent vertebrae in the thoracic spine. There may be a compensatory lumbar lordosis. Symptoms usually settle with postural exercises, and surgical correction is rarely indicated.

'Growing pains' (benign, often nocturnal limb pain) are frequent and occur in approximately $10-20 \%$ of young adolescents. The pain is usually crampy, localised to the lower limbs, occurs in the evening, and can often wake the patient at night. It is, however, never associated with a limp and treatment is symptomatic.

Sports injuries are more frequent in adolescence. For example, boys who play football are more likely to get groin strain and knee and ankle injuries. Anterior knee pain may represent part of a hypermobility syndrome and should always be sought.

Chondromalacia patellae usually affects women and is a malalignment disorder of the patellofemoral apparatus. Symptoms usually include pain on climbing stairs or with any activities which require knee flexion. Although symptoms usually improve with time, a programme of isometric exercises to strengthen the quadriceps may be helpful.

Osgood-Schlatter disease is more common in boys and is due to osteochondritis of the tibial tuberosity. Pain and swelling usually occurs around the tibial tubercle and is aggravated by exercise.

In a proportion of individuals a spiral of chronic musculoskeletal pain can develop for which no underlying cause can be found. It is a condition which is important to recognise, as misdiagnosis and overinvestigation can contribute to the prolongation of the symptoms. ${ }^{20}$ It is useful to consider musculoskeletal pain as being either a localised idiopathic pain syndrome (LIPS) or a diffuse idiopathic pain syndrome (DIPS), and the differential diagnosis is wide. LIPS has been equally reported in boys and girls and usually affects the lower limb. The affected limb is often exquisitely tender and may be held fixed and immobile due to pain. In one study, about $50 \%$ of adolescents with LIPS also had evidence of reflex sympathetic dystrophy with changes of local vasomotor and autonomic dysfunction. ${ }^{21}$ The same study also showed an increased incidence of fibromyalgia in patients with DIPS. In these patients it was noted that there were increased levels of 'stress' in their lives, for example it was common for them to be overachievers or underachievers at school, or to originate from single parent families. A history of sexual abuse was also identified in some individuals. In addition to diffuse pain and stiffness, patients with juvenile fibromyalgia syndrome have also been found to have poor sleep, fatigue, anxiety, and a subjective sensation of soft tissue swelling, compared with normal controls. ${ }^{22}$ There are also studies suggesting that patients with fibromyalgia may have joint hypermobility. ${ }^{23}$

The treatment of chronic idiopathic pain can be difficult. Education of both the parent and child is important and mobility should be improved using a graded programme of exercise. Psychological intervention has an important role.

\section{Management issues}

As adolescents with chronic illness and disability have specific needs, they are best managed by physicians who have a special interest and expertise in this area. The medical transition from a paediatric to an adult system should be a gradual process, which is planned ideally from the time of diagnosis. ${ }^{24}$ During this period adolescents need to be taught more about their illness and disability so that realistic goals are created as they strive towards achieving independence. Important issues regarding education, work experience, and career choices need to be addressed and the family should be encouraged to allow the adolescent to have control in such decision making. The doctor/ patient relationship, which was previously more oriented towards the parent, now needs to be oriented towards the adolescent. This may involve allowing the adolescent to attend clinic appointments alone and respecting their confidentiality. The adolescent should feel that their opinion has been taken into consideration when planning future treatments and they should also be held responsible for compliance with their medication. Finally adolescents need to be encouraged to engage in social activities. As more mature relationships develop, the sensitive issue of sexuality may need to be explored.

It is the role of the physician, as well as the other members of the multidisciplinary team, to work together with the family to ensure that the child progresses as smoothly as possible through adolescence. The transition should be planned and prepared so that the individual reaches early adulthood better equipped to deal with life's challenges and spared the suffering of adolescence coupled with rheumatic disease.

\section{Summary}

Adolescence is a period of emotional and physical upheaval and a time when personal identity, need for independence, and peer relationships all evolve. This transition from dependence to independence can be a difficult process for healthy teenagers. Those with chronic illness and disability have additional concerns, rendering them especially vulnerable as they progress into adulthood.

We wish to thank Mrs Hilary Cook for her secretarial help. 
1 Symmons D, Jones M, Osborne J, et al. Paediatric rheumatology in the United Kingdom: data from the British Paediatric Rheumatology Group Nat

2 Scholz S, Albert ED. Immunogenetics of juvenile chronic arthritis: Clin Exp Rheumatol 1993:11(suppl):S37-41.

3 Leak AM. Management of arthritis in adolescence. $\mathrm{Br} f$ Rheumatol 1994;33:882-8.

4 David J, Cooper C, Hickey L, et al. The functional and psychological outcomes of juvenile chronic arthritis in young adulthood. BrF Rheumatol 1994;33:876-81.

5 Wallace CA, Levinson JE. Juvenile rheumatoid arthritis: outcome and treatment for the 1990s. Rheum Dis Clin North Am 1991;17:891-905.

6 Giannini EH, Brewer EJ, Kuzmina N, et al. Methotrexate in resistant juvenile rheumatoid arthritis. Results of the USAUSSR double blind, placebo controlled trial. $N$ Engl $\mathcal{F} \mathrm{Med}$ 1992;326:1043-9.

7 Wallace CA, Bleyer WA, Sherry DD. Toxicity and serum levels of methotrexate in children with juvenile rheumatoid levels of methotrexate in children with juveni
arthritis. Arthritis Rheum 1989;32:677-81.

8 White PH, Ansell BA. Methotrexate for juvenile chronic arthritis. N Engl f Med 1992;326:1077-8.

9 Rooney $M$. Is there a disease modifying drug for juvenile chronic arthritis? Br $\mathcal{F}$ Rheumatol 1992;31:635-41.

10 Wilkinson VA. Juvenile chronic arthritis in adolescence: facing the reality. Int Rehab Med 1981;3:11-7.

11 Burgos-Vargas R. Spondyloarthropathies + psoriatic arthritis in children. Curr Opin Rheumatol 1993;5:634-43.

12 Southwood TR, Petty RE, Malleson PN, et al. Psoriatic arthritis in children. Arthritis Rheum 1989;32:1007-13.

13 Ansell BM. Juvenile psoriatic arthritis. Ballieres Clin Rheumatol 1994;8:317-32.
14 Silverman ED, Eddy A. Systemic lupus erythematosus in childhood and adolescence. In: Maddison PJ, Isenberg DA, Woo P, Glass Oxford. Oxford University Press, 1993:756-71.

15 Cassidy J. Systemic lupus erythematosus, juvenile dermatomyositis, scleroderma and vasculitis. In: Kelly W, Harris E, Ruddy S, Sledge C, eds. Textbook of rheumatology. Philadelphia: WB Saunders, 1993:1224-8.

16 Gonzalez-Crespo JJ, Gomez-Reino R, Merino R, et al. Menstrual disorders in girls with systemic lupus erythematosus treated with cyclophosphamide. Br f Rheumatol 1995;34: $737-41$

17 Abu-Shakira M, Gladman DD, Urowitz MB. Malignancy and SLE. Arthritis Rheum 1996;39:1050-4.

18 Dillon MJ, Ansell BA. Vasculitis in children and adolescents. Rheum Dis Clin North Am 1995;21:1115-36.

19 Hollingworth P. Back pain in children. Br f Rheumatol 1996; 35:1022-8.

20 Southwood TR. Recent developments in the understanding of paediatric musculoskeletal pain syndromes. Ann Rheum Dis 1993;52:490-2.

21 Mallerson PN, Al-Mater M, Petty GC. Idiopathic musculoskeletal pain syndromes in children. $\mathcal{f}$ Rheumatol 1992;19: 1786-9

22 Yunus MB, Masi AT. Juvenile primary fibromyalgia syndrome. A clinical study of 33 patients and matched normal controls. Arthritis Rheum 1985;28:138-45.

23 Gedalia A, Press J, Klein M, Buskila D. Joint hypermobility and fibromyalgia in school children. Ann Rheum Dis 1993; 52:494-6.

24 White PH. Future expectations: adolescents with rheumatic diseases and their transition into adulthood. Brf Rheumatol 1996;35:80-3. 